\title{
Water Wave Equation Arising On Logarithmic Quantum Mechanics
}

\author{
Hajrulla $\mathrm{Sh}^{1}$, Bezati $\mathrm{L}^{2} \& . H o x h a \mathrm{~F}^{2}$ \\ ${ }^{1}$ University of Vlora, Albania \\ University of Tirana, Albania ${ }^{2}$
}

\begin{abstract}
:
We introduce the model of equation that is closely related to the following equation (1.1). We study the global existence of week solution for this class of equation. Using the Gross logarithmic Sobolev inequality we establish the main theorem of existence of week solution for this class of equation arising from Logarithmic Quantum Mechanics. We can extend the results of $[1,2]$
\end{abstract}

Keywords: logarithmic quantum mechanics; Gross-Sobolev inequality; logarithmic wave equation; global existence of solution; nonlinear effects.

\section{Introduction}

We deal with a mathematical analysis for the problem of water wave equation on Logarithmic quantum mechanics. The main difference between our work and [1,2] is: our problem is in $k$ dimensional case on $H_{0}{ }^{m}$ and involves another nonlinear term $u \log |u|^{k}$; there is no restrictions on the coefficient of the logarithmic nonlinear termu log $|u|^{k}$. Recently in [8] a numerical model is given.

We mainly establish the global existence of weak solutions to the problem (1.2). Firstly we write the problem in a weak version. Secondly we construct approximate solutions by the Galerkin method. Finally we prove the convergence of the sequence of the approximate solutions. To get a priori estimates of the approximate solutions, we employ the Gross logarithmic Sobolev inequality and logarithmic Gronwall inequality.

We introduce the model of equation that is closely related to the following equation with logarithmic nonlinearity. This type of equation arising from many applications in many branches of physics such as nuclear physics, optics and geophysics [7, 9, 10].

$$
\begin{array}{cr}
u_{t t}-(\Delta)^{2} u+u-\varepsilon \log |u|^{2}+|u|^{2} u=0, & (x, t) \in \mathrm{Q} \times(0, \mathrm{~T}) \\
u(x, t)=0, & (x, t) \in \partial \mathrm{Q} \times(0, \mathrm{~T}) \\
u(x, 0)=u^{0}(x) \text { and } u_{t}(x, 0)=u^{1}(x), & x \in \Omega,
\end{array}
$$

where $Q$ is a finite interval $[\mathrm{a}, \mathrm{b}]$, the parameter $\varepsilon$ measures the force of the nonlinear interaction and the nonlinear effects in quantum mechanics are very small. The problem (1.1) is a relativistic version of logarithmic quantum mechanics introduced in $[3,4]$.

In this paper we deal with global existence of week solutions for the initial boundary value problem of the logarithmic wave equation:

$$
\begin{array}{lr}
u_{t t}-A u+u+u_{t}-u \log |u|^{k}+|u|^{k} u=0, & (x, t) \in \Omega \times(0, \mathrm{~T}) \\
u(x, t)=0, & (x, t) \in \partial \Omega \times(0, \mathrm{~T})
\end{array}
$$




$$
u(x, 0)=u^{0}(x) \text { and } u_{t}(x, 0)=u^{1}(x)
$$

$x \in \Omega$,

where $\quad \Omega \subset R^{n}, \mathrm{n} \geq 1, \quad$ is a bounded domain with smooth boundary $\partial \Omega, \mathrm{k} \geq 1$ is an integer and $A=(\Delta)^{m},(m \geq 1$ is a parameter $)$. Here $u$ is a complex scalar field. The model (1.2) is introduced in [8] for studying the dynamics of Q-ball in theoretical physics. The logarithmic nonlinearity is of much interest in physics, since it appears naturally in cosmology and symmetric filed theories, quantum mechanics and nuclear physics $[1,14]$. This type of problems have many applications in many branches of physics such as nuclear physics, optics and geophysics. It has been also introduced in the quantum field theory.

In [1]- [2], Cazenave and Haraux established the existence a solution for the following equation

$$
u_{t t}+A+u+u_{t}|u|^{2} u=u \ln |u|, \quad x \in \Omega, \mathrm{t}>0
$$

for studying the dynamics of Q-ball in theoretical physics. In [2], Cazenave and Haraux established the existence and uniqueness of a solution for the Cauchy problem for the following equation in $R^{n}$.

$$
u_{t t}+A=u l n|u|^{k} \text {, }
$$

In the following section we state some lemmas. In the section 3 we give the proof of the theorem.

\section{Preliminaries for the Theorem of existence of week solution}

We denote by $\|.\|_{p}$ the $\mathrm{L}^{\mathrm{p}}(\Omega)$ norm, and $\|\nabla$.$\| the Dirichlet norm in H_{0}{ }^{m}$. In particular, we denote $\|$. $=\|.\|_{2}$. We also use $C$ to denote a universal positive constant that may have different values in different places. We denote by $(\cdot, \cdot)$ the inner product in $\mathrm{L}^{2}(\Omega)$. and by $\langle\cdot, \cdot\rangle$ the duality pairing between $H_{0}{ }^{1}$ and $H_{0}{ }^{m}$. We also use $\mathrm{C}$ to denote a universal positive constant may take different values in different places. Let's we introduce the definition of weak solutions for the problem (1.2).

Def. A function $u$ on $[0, T]$ is called a weak solution $u(x, t)$ of problem (1.2) on $\Omega \times[0$, T), if $u \in$ $C\left([0, T], H_{0}{ }^{m}(\Omega)\right), \mathrm{u}^{\prime} \in C\left([0, T], \mathrm{L}^{2}(\Omega)\right), u_{0}=u^{0}(x)$ and $\mathrm{u}^{\prime}(0)=u^{1}(x)$ and $u$ satisfies,

$$
\left\langle\mathrm{u}^{\prime \prime}(\mathrm{t}), \emptyset\right\rangle+(\nabla u, \nabla \emptyset)+(u, \emptyset)+\left(\mathrm{u}^{\prime}, \emptyset\right)-\left(u \log |u|^{k}, \varnothing\right)+\left(|u|^{k} u, \emptyset\right)=0
$$

Lemma 2.1: (See [12]) Assume $v \in H_{0}{ }^{m}(\Omega), \quad$ and $\Omega$ is a bounded smooth domain in $R^{n}\left(\Omega \subset R^{n}\right)$. Then, for any $a>0$, it holds that

$$
\int_{\Omega}|v|^{2} \log |v| d x \leq\left(\frac{3}{4} \log \frac{4 a}{e}\right)\|v\|_{2}^{2}+\frac{a}{4}\|\nabla v\|_{2}^{2}+\|v\|_{2}^{2} \log \|v\|_{2}
$$

Lemma 2.2: (See [13]) Assume $w(t)$ is nonnegative, $w(t) \in \mathrm{L}^{\infty}(0, \mathrm{~T}), w(0)>0$ and

$$
w(t) \leq w(0)+a \int_{\Omega} w(s) \log [a+w(s)] d s \quad t \in(0, \mathrm{~T}),
$$

where $a>1$ is a positive constant. Then we have $w(t) \leq(a+w(0))^{\mathrm{e}^{\text {at }}}, t \in(0, \mathrm{~T})$,

Lemma 2.3: (see [3, 5]) (Logarithmic Sobolev inequality) Let $\boldsymbol{u}$ be any function in $H_{0}{ }^{m}(\Omega)$, and $a>0$ be any number. Then 


$$
\begin{array}{r}
2 \int_{\Omega}|u|^{2} \ln \frac{|u|}{\|u\|} d x+n(1+\log a)\|u\|^{2} \\
\leq \frac{a^{2}}{\pi} \int_{\Omega}\left|\frac{1}{2} u\right|^{2} d x
\end{array}
$$

\section{The main theorem and the proof}

In this section we deal with main theorem of existence of global week solution. By using these lemmas and using the Gross logarithmic Sobolev inequality with the combination of Galerkin method to construct approximate solutions, we can proof the main theorem. We carry out the proof of Theorem giving the solution $\boldsymbol{u}$, where $\boldsymbol{u}$ is a weak solution of problem (1.2) on [0,T ), where $T$ is the maximal existence time of weak solution The proof is based on Galerkin method. We use the Gross logarithmic Sobolev inequality and the logarithmic Gronwall inequality.

Theorem 3.1 Assume that $u^{0}(x) \in H_{0}{ }^{m}(\Omega)$, and $u^{1}(x) \in \mathrm{L}^{2}(\Omega)$. Then, the problem (1.2) admits global weak solution defined on $[0, T]$ for any $T>0$.

Proof: Let $\left\{w_{j}\right\}_{j=1}^{\infty}$ be the eigenfunctions of the operator $A=(\Delta)^{m}$ with zero Dirichlet boundary condition and $D(A)=H_{0}{ }^{m}(\Omega) \cap H_{0}{ }^{1}(\Omega)$. It is well-known that $\left\{w_{j}\right\}_{j=1}^{\infty}$ forms an orthonormal basis for $\mathrm{L}^{2}(\Omega)$ as well as for $H_{0}{ }^{1}(\Omega)$. Let $P_{k}$ be the orthogonal projection of $\mathrm{L}^{2}(\Omega)$ onto $V_{k}=$ the linear span of $\left\{w_{1}, \ldots, w_{k}\right\}, k \geq 1$. Let $u_{k}=\sum_{j=1}^{k} g_{k_{j}}(t) w_{j}$ be an approximate solution to (1.2) in $V_{k}$. Then $u_{k}(t)$ verifies the following system of ODEs:

$$
\begin{gathered}
\left\langle\mathrm{u}_{k}{ }_{k}(\mathrm{t}), w_{j}\right\rangle+\left(\nabla u_{k}(t), \nabla w_{j}\right)+\left(u_{k}, w_{j}\right)+\left(\mathrm{u}^{\prime}{ }_{k}(\mathrm{t}), w_{j}\right)-\left(u_{k} \log \left|u_{k}\right|^{2}, w_{j}\right)+\left(\left|u_{k}\right|^{2} u_{k}, w_{j}\right) \\
=0 \\
u_{k}(0)=P_{k} u^{0}(x), \quad \mathrm{u}^{\prime}{ }_{k}(0)=P_{k} u^{1}(x)
\end{gathered}
$$

for $j=1, \ldots, k$. More specifically,

$$
u_{k}(0)=\sum_{j=1}^{k} u_{k_{j}}(0) w_{j}, \quad \mathrm{u}^{\prime}{ }_{k}(0)=\sum_{j=1}^{k} \mathrm{u}^{\prime}{ }_{k_{j}}(0) w_{j}
$$

where,

$$
u_{k_{j}}(0)=\left(u^{0}, w_{j}\right), \quad \mathrm{u}^{\prime}{ }_{k_{j}}(0)=\left(u^{1}, w_{j}\right), \quad j=1, \ldots, k
$$

Obviously, $u_{k}(0) \rightarrow u^{0}$ strongly in $H_{0}{ }^{m}(\Omega), \mathrm{u}^{\prime}{ }_{k}(0) \rightarrow u^{1}$ strongly in $\mathrm{L}^{2}(\Omega)$ as $k \rightarrow \infty$. By using the Cauchy-Peano theorem, we know that the system (3.1)-(3.2) admits a solution $g_{k_{j}}(\mathrm{t}) \in \mathrm{C}^{2}\left[0, T_{k}\right]$ for every $k \geq 1$ and some $T_{k}>0$. Then we can obtain an approximate solution $u_{k}(t)$ of the problem (1.2) over $\left[0, T_{k}\right]$. Now we try to get the a priori estimate for the approximate solutions $u_{k}(t)$ of the problem (1.2). Multiplying (3.1) by ${ }^{\prime}{ }_{k_{j}}(t)$ and summing with respect to $j$ from 1 to $k$, we have

$$
=0 \quad \frac{\mathrm{d}}{\mathrm{dt}}\left[\frac{1}{2}\left\|\mathrm{u}^{\prime}{ }_{k}(\mathrm{t})\right\|_{2}^{2}+\left\|u_{k}(t)\right\|_{2}^{2}-\int_{\Omega}\left|u_{k}(t)\right|^{2} \log \left|u_{k}(t)\right| d x+\frac{1}{4}\left\|u_{k}(t)\right\|_{4}^{4}\right]+\left\|\mathrm{u}_{k}{ }_{k}(\mathrm{t})\right\|_{2}^{2}
$$

Integrating (3.3) over $(0, t), 0<t \leq T_{k}$, we get 


$$
\begin{aligned}
& \frac{1}{2}\left\|\mathrm{u}^{\prime}{ }_{k}(\mathrm{t})\right\|_{2}^{2}+\frac{1}{2}\left\|\nabla u_{k}(t)\right\|_{2}^{2}+\left\|u_{k}(t)\right\|_{2}^{2}-\int_{\Omega}\left|u_{k}(t)\right|^{2} \log \left|u_{k}(t)\right| d x+\frac{1}{4}\left\|u_{k}(t)\right\|_{4}^{4} \\
& \quad+\int_{0}^{t}\left\|\mathrm{u}^{\prime}{ }_{k}(\mathrm{~s})\right\|_{2}^{2} d s \\
& \quad=\frac{1}{2}\left\|\mathrm{u}^{\prime}{ }_{k}(0)\right\|_{2}^{2}+\frac{1}{2}\left\|\nabla u_{k}(0)\right\|_{2}^{2}+\left\|u_{k}(0)\right\|_{2}^{2}-\int_{\Omega}\left|u_{k}(0)\right|^{2} \log \left|u_{k}(0)\right| d x+\frac{1}{4}\left\|u_{k}(0)\right\|_{4}^{4} \\
& \quad+\int_{0}^{t}\left\|\mathrm{u}^{\prime}{ }_{k}(\mathrm{~s})\right\|_{2}^{2} d s \\
& \leq C_{0}-\int_{\Omega}\left|u_{k}(0)\right|^{2} \log \left|u_{k}(0)\right| d x
\end{aligned}
$$

where $C_{0}=C\left(\left\|u^{0}\right\|_{\mathrm{H}^{\mathrm{m}}(\Omega)},\left\|u^{1}\right\|_{\mathrm{L}^{2}(\Omega)}\right)$ is a positive constant. We use the elementary inequality

$$
\begin{aligned}
& \left|\mathrm{t}^{2} \log \mathrm{t}\right| \leq \mathrm{C}\left(1+\mathrm{t}^{3}\right) \quad \text { for all } t>0 \\
& -\int_{\Omega}\left|u_{k}(0)\right|^{2} \log \left|u_{k}(0)\right| d x \leq \mathrm{C}|\Omega|+C \int_{\Omega}\left|u_{k}(0)\right|^{3} d x \leq \mathrm{C}\left(1+\left\|u_{k}(0)\right\|_{\mathrm{H}^{\mathrm{m}}(\Omega)}^{3}\right) \\
& \quad \leq \mathrm{C}(1 \\
& \left.\quad+\left\|u^{0}\right\|_{\mathrm{H}^{\mathrm{m}}(\Omega)}^{3}\right)
\end{aligned}
$$

From (3.4) and (3.6) we have

$$
\frac{1}{2}\left\|\mathrm{u}^{\prime}{ }_{k}(\mathrm{t})\right\|_{2}^{2}+\frac{1}{2}\left\|\nabla u_{k}(t)\right\|_{2}^{2}+\left\|u_{k}(t)\right\|_{2}^{2}+\frac{1}{4}\left\|u_{k}(t)\right\|_{4}^{4}+\int_{0}^{t}\left\|\mathrm{u}^{\prime}{ }_{k}(\mathrm{~s})\right\|_{2}^{2} d s
$$

$\leq C+\int_{\Omega}\left|u_{k}(t)\right|^{2} \log \left|u_{k}(t)\right| d x$

Now we use Lemma 2.1 introducing Gross-Sobolev inequality and we have

$$
\begin{gathered}
\int_{\Omega}\left|u_{k}(t)\right|^{2} \log \left|u_{k}(t)\right| d x \\
\leq\left(\frac{3}{4} \log \frac{4 \mathrm{a}}{\mathrm{e}}\right)\left\|u_{k}(t)\right\|_{2}^{2}+\frac{\mathrm{a}}{4}\left\|\nabla u_{k}(t)\right\|_{2}^{2}+\left\|u_{k}(t)\right\|_{2}^{2} \log \left\|u_{k}(t)\right\|_{2}
\end{gathered}
$$

From (3.7) and (3.8), we take $a=\frac{1}{4}$ and we obtain

$$
\begin{aligned}
\frac{1}{2}\left\|\mathrm{u}^{\prime}{ }_{k}(\mathrm{t})\right\|_{2}^{2} & +\left(\frac{1}{2}-\frac{\mathrm{a}}{4}\right)\left\|\nabla u_{k}(t)\right\|_{2}^{2}+\left(1-\frac{3}{4} \log \frac{4 \mathrm{a}}{\mathrm{e}}\right)\left\|u_{k}(t)\right\|_{2}^{2}+\frac{1}{4}\left\|u_{k}(t)\right\|_{4}^{4}+\int_{0}^{t}\left\|\mathrm{u}^{\prime}{ }_{k}(\mathrm{~s})\right\|_{2}^{2} d s \\
& \leq C \\
& +\left\|u_{k}(t)\right\|_{2}^{2} \log \left\|u_{k}(t)\right\|_{2}
\end{aligned}
$$




$$
\begin{gathered}
\left\|\mathrm{u}^{\prime}{ }_{k}(\mathrm{t})\right\|_{2}^{2}+\left\|\nabla u_{k}(t)\right\|_{2}^{2}+\left\|u_{k}(t)\right\|_{2}^{2}+\left\|u_{k}(t)\right\|_{4}^{4} \\
\leq C\left(1+\left\|u_{k}(t)\right\|_{2}^{2} \log \left\|u_{k}(t)\right\|_{2}\right)
\end{gathered}
$$

$$
\begin{aligned}
& \left\|u_{k}(t)\right\|_{2}^{2} \leq 2\left(\left\|u_{k}(0)\right\|_{2}^{2}+2 T \int_{0}^{t}\left\|\mathrm{u}^{\prime}{ }_{k}(\mathrm{~s})\right\|_{2}^{2} d s\right. \\
& \leq 2\left(\left\|u_{k}(0)\right\|_{2}^{2}\right. \\
& +\max \{1,2 T\} \frac{1+\mathrm{C}}{\mathrm{C}} \int_{0}^{t}\left\|\mathrm{u}^{\prime}{ }_{k}(\mathrm{~s})\right\|_{2}^{2} d s
\end{aligned}
$$$$
\text { Note that } u_{k}(t)=u_{k}(0)+\int_{0}^{t} \mathrm{u}^{\prime}{ }_{k}(\mathrm{~s}) d s, \quad \text { than we have }
$$

From (3.10) and (3.11) we have

$$
\left\|u_{k}(t)\right\|_{2}^{2} \leq A+B \int_{0}^{t}\left\|u_{k}(s)\right\|_{2}^{2} \log \left\|u_{k}(s)\right\|_{2} d s
$$

where $A=\left\|u_{k}(0)\right\|_{2}^{2}+\max \{1,2 T\}$ and $B=\max \{1,2 T\}(1+C)$. Taking $B \geq 1$ and using the Gronwall inequality in Lemma 2.2 we get

$$
\begin{gathered}
\left\|u_{k}(t)\right\|_{2}^{2} \leq A+B \int_{0}^{t}\left\|u_{k}(s)\right\|_{2}^{2} \log \left\|u_{k}(s)\right\|_{2} d s \\
\left\|u_{k}(t)\right\|_{2}^{2} \leq(A+B)^{\mathrm{e}^{\mathrm{Bt}}} \leq C_{T}
\end{gathered}
$$

From (3.10) and (3.13)

$$
\left\|\mathrm{u}^{\prime}{ }_{k}(\mathrm{t})\right\|_{2}^{2}+\left\|\nabla u_{k}(t)\right\|_{2}^{2}+\left\|u_{k}(t)\right\|_{2}^{2}+\left\|u_{k}(t)\right\|_{4}^{4} \leq C_{T}
$$

Note that $u_{k}$ is uniformly bounded in $\mathrm{L}^{\infty}\left(0, T, H_{0}{ }^{m}(\Omega)\right), \mathrm{u}^{\prime}{ }_{k}$ is uniformly bounded in $\mathrm{L}^{\infty}\left(0, T, L^{2}(\Omega)\right)$, $\mathrm{u}^{\prime \prime}{ }_{k}$ is uniformly bounded in $\mathrm{L}^{\infty}\left(0, T, H_{0}{ }^{-m}(\Omega)\right)$. From these refers and using (3.14), there exist a subsequence $\left\{u_{k}\right\}$, such that implies the system (3.15) as follow:

$$
\begin{aligned}
& u_{k} \rightarrow u \quad \text { weekly in } \mathrm{L}^{\infty}\left(0, T, H_{0}{ }^{m}(\Omega)\right), \\
& \underset{\mathrm{u}^{\prime}{ }_{k} \rightarrow \mathrm{u}^{\prime} \quad \text { weekly in } \mathrm{L}^{\infty}\left(0, T, L^{2}(\Omega)\right),}{(3.15)} \\
& \mathrm{u}^{\prime \prime}{ }_{k} \rightarrow \mathrm{u}^{\prime \prime} \quad \text { weekly in } \mathrm{L}^{\infty}\left(0, T, H_{0}{ }^{-m}(\Omega)\right)
\end{aligned}
$$

Using these refers as above we have

$$
\begin{aligned}
& u_{k} \rightarrow u \quad \text { strongly in } \mathrm{L}^{2}\left(0, T, \mathrm{~L}^{2}(\Omega)\right) \\
& \left.u_{k} \rightarrow u \quad \text { in }(0, T) \times \Omega\right) \\
& (0, T) \times \Omega) \\
& \left.\left|u_{k}\right|^{2} u_{k} \rightarrow|u|^{2} u \quad \text { in }(0, T) \times \Omega\right)
\end{aligned}
$$




$$
\int_{\Omega}\left|u_{k} \log u_{k}{ }^{2}\right|^{2} d x=4 \int_{\Omega}\left|u_{k}\right|^{2}\left(\log u_{k}{ }^{2}\right) d x \leq C|\Omega|+C \int_{\Omega}\left|u_{k}\right|^{2} d x \leq C|\Omega|\left(\left\|u_{k}(s)\right\|_{H_{0}}^{6} m+1\right) \leq C
$$

This implies that $u_{k} \log \left|u_{k}\right|^{2}{ }_{k}$ is uniformly bounded in $\mathrm{L}^{2}\left(0, T, \mathrm{~L}^{2}(\Omega)\right)$. So, exists any function in $\mathrm{L}^{2}\left(0, T, \mathrm{~L}^{2}(\Omega)\right)$, such that $\left|u_{k}\right|^{2} u_{k}$ converges in it. This is $u \log |u|^{2}$. So,

So,

$$
u_{k} \log \left|u_{k}\right|^{2} \rightarrow u \log |u|^{2} \quad \text { in } \mathrm{L}^{2}\left(0, T, \mathrm{~L}^{2}(\Omega)\right)
$$

By Sobolev inequality

$$
\leq\left.\left. C \quad \int_{\Omega}|| u_{k}\right|^{2} u_{k}\right|^{2} d x=\int_{\Omega}\left|u_{k}\right|^{6} \leq C\left\|u_{k}(s)\right\|_{H_{0}}^{6} m
$$

As above we explained there exist any function as $|u|^{2} u$ in $L^{2}\left(0, T, L^{2}(\Omega)\right)$ such that

$$
\begin{aligned}
& \left|u_{k}\right|^{2} u_{k} \rightarrow|u|^{2} u \quad \text { in } \mathrm{L}^{2}\left(0, T, \mathrm{~L}^{2}(\Omega)\right) \\
& (3.21)
\end{aligned}
$$

Using (3.18), (3.19), (3.20) in (3.1) and passing to the limit in (3.1) and since $\left\{u_{k}\right\}_{j=1}^{\infty}$ is dense in $H_{0}{ }^{m}(\Omega)$

$$
\begin{aligned}
& \left\langle u^{\prime \prime}, w\right\rangle+(\nabla u, \nabla v)+\left(u, w_{j}\right)+\left(u^{\prime}, v\right)-\left(u \log |u|^{2}, v\right)+\left(|u|^{2} u, v\right) \\
& =0 \quad v \in H_{0}{ }^{m}(\Omega)
\end{aligned}
$$

This clearly said that $u$ satisfies the eq. (1.2) in the week sense. From (3.15) we have $u_{k}(0) \rightarrow u(0)$ weekly in $\mathrm{L}^{2}(\Omega)$. Using eq.3.16 and by choosing $u_{k}(0) \rightarrow u^{0}$ strongly in $H_{0}{ }^{m}(\Omega)$, we have

$$
u(0)=u^{0}
$$

From (3.16), $\left\langle u{ }^{\prime \prime}{ }_{k}, w_{j}\right\rangle \rightarrow\left\langle u, w_{j}\right\rangle$ in $\mathrm{L}^{\infty}(0, T)$. This implies that $\left\langle u^{\prime}{ }_{k}(0), w_{j}\right\rangle \rightarrow\left\langle\mathrm{u}^{\prime}(0), w_{j}\right\rangle$. Noting that $u^{\prime}{ }_{k}(0) \rightarrow u^{1}$ weekly in $\mathrm{L}^{2}$, than

$$
u^{\prime}(0)=u^{1}
$$

This completes the theorem (3.1) and the initial condition is satisfied.

\section{References}

[1] Hiramatsu T., Kawasaki M. and Takahashi F., Numerical study of Q-ball formation in gravity mediation. Journal of Cosmology and Astroparticle Physics, 2010(6) (2010), 001-008.

[2] Cazenave T., Haraux A., Equations d'evolution avec non-linearite logarithmique. Ann. FacSci. Toulouse Math., 2(1) (1980), 21-51.

[3] Gorka P., Logarithmic Klein-Gordon equation. Acta Phys. Polon. B, 40(1) (2009), 59-66.

[4] Bialynicki-Birula I,Mycielski J, Nonlinear wave mechanics. Ann. Physics, 100(1-2) (1976), 62-93.

[5] Gross L., Logarithmic Sobolev inequalities. Amer. J. Math., 97(4) (1975), 1061-1083.

[6] Enzo Vitillaro. Global existence for the wave equation with nonlinear boundary damping and source terms. J. Differential Equations , 186(1):259-298, 2002.

[7] H. Buljan, A. Siber, M. Soljacic, T. Schwartz, M. Segev, and D. N. Christodoulides, Incoherent white light solitons in logarithmically saturable noninstantaneous nonlinear media, Phys. Rev. E (3) 68 (2003), no. 3, 036607, 6 pp. 
[8] T. Hiramatsu, M. Kawasaki, and F. Takahashi, Numerical study of Q-ball formation in gravity mediation, Journal of Cosmology and Astroparticle Physics 2010 (2010), no. 6, 008.

[9] S. De Martino, M. Falanga, C. Godano, and G. Lauro, Logarithmic Schr“odinger-like equation as a model for magma transport, Europhys. Lett. 63 (2003), no. 3, 472-475.

[10] W. Krolikowski, D. Edmundson, and O. Bang, Unified model for partially coherent solitons in logarithmically nonlinear media, Phys. Rev. E 61 (2000), 3122-3126.

[11] P. Gorka, Logarithmic Klein-Gordon equation, Acta Phys. Polon. B 40 (2009), no. 1, 59-66.

[12] L. Gross, Logarithmic Sobolev inequalities, Amer. J. Math. 97 (1975), no. 4, 1061-1083.

[13] T. Cazenave and A. Haraux, Equations d'evolution avec non-lin'earit'e logarithmique, Ann. Fac. Sci. Toulouse Math. (5) 2 (1980), no. 1, 21-51.

[14] K. Enqvist and J. McDonald, Q-balls and baryogenesis in the MSSM, Phys. Lett. B 425 (1998), 309321. 